\title{
Therapy-associated myelodysplastic syndrome with monosomy 7 arising in a Muir-Torre Syndrome patient carrying SETBP1 mutation
}

\author{
DAVID ULLMAN $^{1 *}$, ERIN BAUMGARTNER ${ }^{1 *}$, NICHOLAS WNUKOWSKI $^{1}$, GABE KOENIG $^{1}$, \\ FADY M. MIKHAIL ${ }^{3}$, PETER PAVLIDAKEY ${ }^{2}$ and DENIZ PEKER ${ }^{1}$
}

Departments of ${ }^{1}$ Pathology, ${ }^{2}$ Dermatology and ${ }^{3}$ Genetics, University of Alabama at Birmingham, Birmingham, AL 35233, USA

Received September 13, 2017; Accepted November 30, 2017

DOI: $10.3892 /$ mco.2017.1532

\begin{abstract}
Muir-Torre Syndrome (MTS) is a rare hereditary autosomal dominant cancer syndrome and is linked to hereditary non-polyposis colorectal carcinoma (Lynch Syndrome). Individuals develop various skin neoplasms in addition to colorectal, endometrial and upper gastrointestinal malignancies. Therapy-associated myelodysplastic syndrome (T-MDS) is an aggressive hematologic malignancy and is considered a pre-leukemic phase. T-MDS is associated with prior exposure to chemo- and radiotherapy that potentially results in DNA damage. The current case report presents a 74-year-old male MTS patient with prior history of solid tumors and radiation therapy with new onset cytopenia. A subsequent bone marrow biopsy revealed multilineage dysplasia with a high blast count and a diagnosis of high grade T-MDS was rendered. FISH and G-banded karyotype analyses revealed $5 \mathrm{q}$ deletion and monosomy 7. This is a unique case of T-MDS arising in the setting of MTS. Secondary malignancies including MDS and acute leukemia may occur in cancer survivors and are often associated with an unfavorable prognosis. This case demonstrates the need to be aware of the risk of secondary hematologic malignancies in cancer patients and a thorough clinical and lab work-up are warranted in patients with persistent or transfusion requiring cytopenia(s).
\end{abstract}

\section{Introduction}

Hereditary non-polyposis colorectal carcinoma (HNPCC) is an autosomal dominant genetic disorder characterized by

Correspondence to: Dr Deniz Peker, Department of Pathology, University of Alabama at Birmingham, 1802 6th Avenue South, NP 3545, Birmingham, AL 35233, USA

E-mail: dpeker@uabmc.edu

*Contributed equally

Key words: Muir-Torre, MDS, therapy-related, chemotherapy, radiation a deficiency of DNA mismatch repair proteins (1-3). This deficiency leads to the formation of various neoplasms including colorectal carcinoma and malignancies of the endometrium, ovary, stomach, small bowel, and pancreas. Muir-Torre Syndrome (MTS) is subtype of HNPCC characterized by the additional presence of various dermatologic neoplasms including sebaceous adenomas, sebaceous carcinomas, and keratoacanthomas (1-3). HNPCC is not traditionally associated with hematologic malignancies (4).

MDS is defined by the World Health Organization (WHO) as a group of clonal bone marrow neoplasms characterized by ineffective hematopoiesis, manifested by morphologic dysplasia in hematopoietic cells and peripheral cytopenia (5). Therapy-related MDS (t-MDS) is a subtype of MDS defined by the WHO as a distinct category in the classification of patients who develop myeloid neoplasms following cytotoxic therapy (5). Associations between t-MDS and several germline mutations have been established but, to our knowledge, no association between t-MDS and MTS has been reported.

\section{Case report}

Patient and methods. A 74-year-old male with MTS presented with a new onset anemia. Approximately 10 years previously, the patient was treated at an outside hospital for a variety of malignancies including gastric adenocarcinoma, colorectal adenocarcinoma, prostatic adenocarcinoma, and multiple cutaneous neoplasms. The patient reported undergoing several surgical operations including a resection of approximately $70 \%$ of his stomach for gastric adenocarcinoma. While specific details of his treatment are unavailable, the patient reported being treated with fluorouracil (5-FU) and radiation for his gastric adenocarcinoma.

Initial complete blood count revealed a hemoglobin of $7.6 \mathrm{~g} / \mathrm{dl}$, a reticulocyte count of 2.59 , and a platelet count of 95,000 platelets/dl. Given the patient's history and new onset anemia, an evaluation including a peripheral blood smear and bone marrow biopsy was performed. FISH analysis and a hematologic panel of next generation sequencing were performed on the bone marrow specimen. Written informed consent was obtained from the patient. 


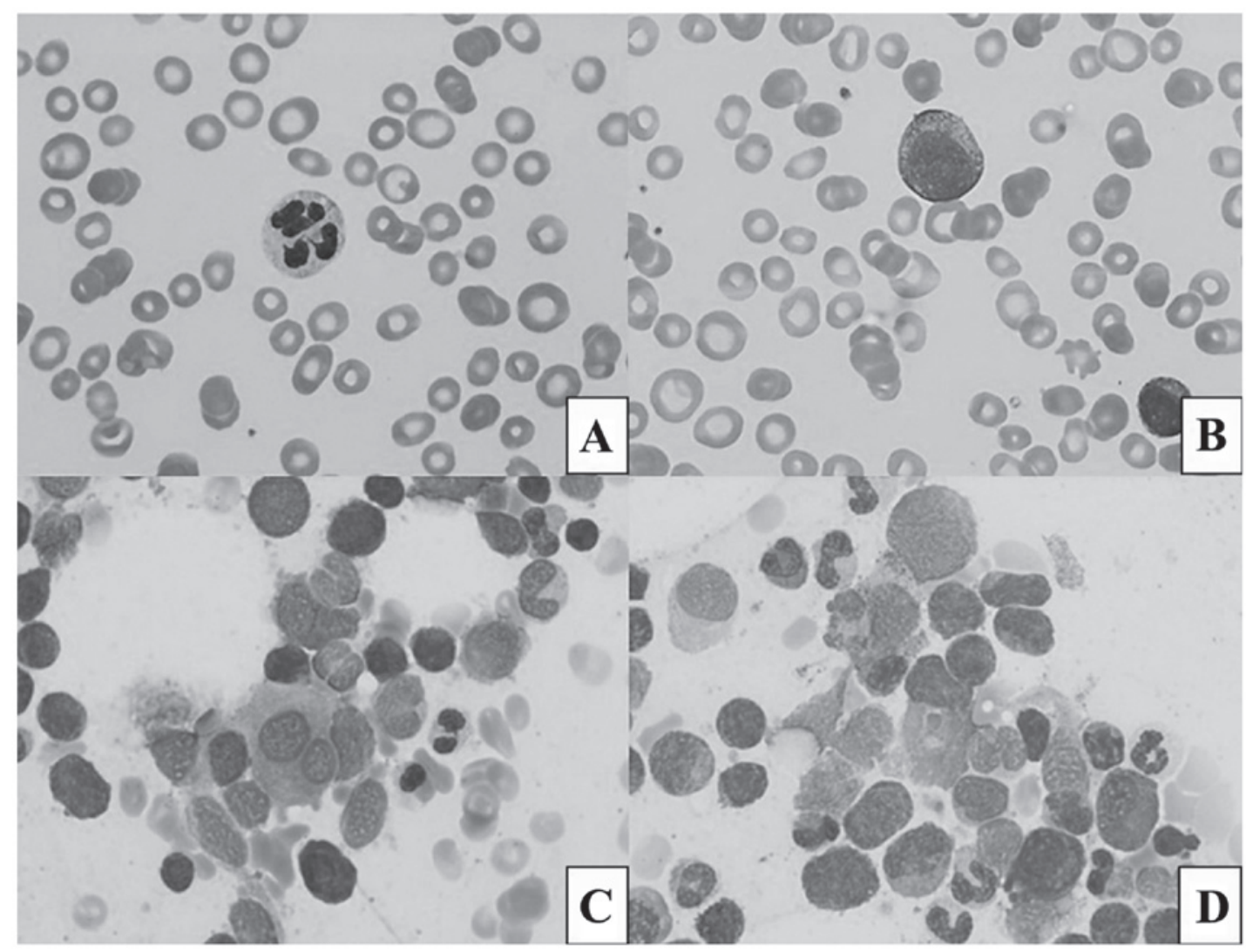

Figure 1. (A) Hypersegmented neutrophil, peripheral blood (Wright-Giemsa, original magnification x1,000). (B) Blast, peripheral blood (Wright-Giemsa, original magnification x1,000). (C and D) Dysplastic changes in all three lineages, bone marrow aspirate (Wright-Giemsa, original magnification x1,000).

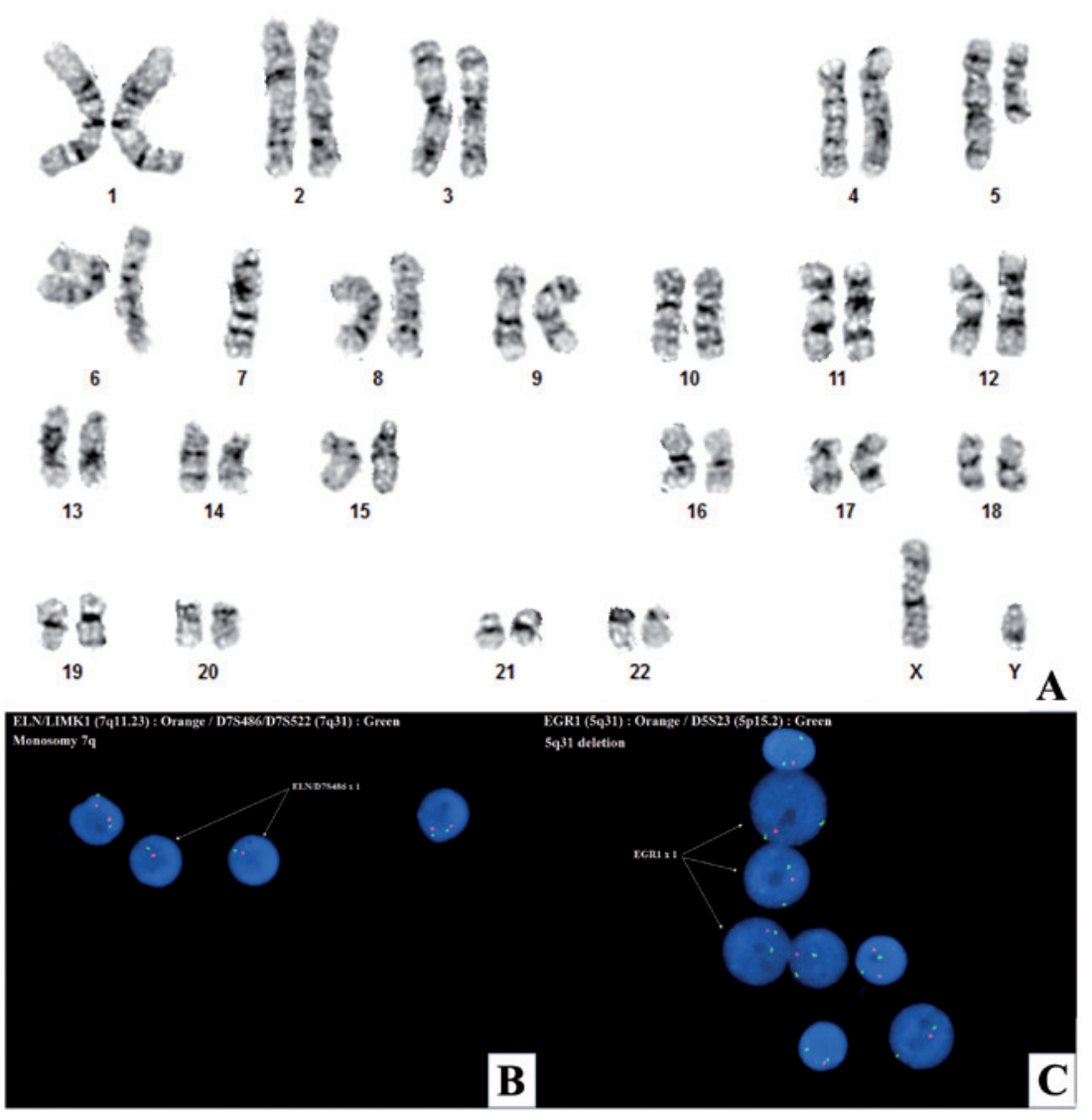

Figure 2. Cytogenetic analysis (A) G-banded karyotype demonstrating 5q13q33 deletion and monosomy 7 [45,XY,del(5)(q13q33),-7], (B) Interphase FISH analysis demonstrating monosomy 7q. Note the deletion of the ELN/LIMK1 orange probe at 7q11.23 and the D7S486/D7S522 green probe at 7q31, (C) Interphase FISH analysis demonstrating $5 \mathrm{q} 31$ deletion. Note the deletion of the EGR1 orange probe at $5 \mathrm{q} 31$. 


\section{Results}

The peripheral blood smear revealed dysplastic, hypo- and hypersegmented neutrophils and rare blasts (Fig. 1A and B). The bone marrow biopsy revealed megakaryocytic, erythroid and myeloid dysplasia (Fig. 1C and D) and concurrent flow cytometry revealed an increased portion of blasts (12\%). FISH analysis (Fig. 2), revealed $5 \mathrm{q} 31$ deletion and monosomy $7 \mathrm{q}$ in $\sim 88 \%$ of interphase cells (Fig. 2B and C). G-banded chromosome analysis demonstrated an interstitial $5 q 13 q 33$ deletion and monosomy 7 in all 20-metaphase cells examined [45,XY,del(5) (q13q33),-7] (Fig. 2A). A hematologic panel of next generation sequencing was performed on the bone marrow revealing mutations in SETBP1 p.G870S and NF1 p.S340Cfs"12. The patient was diagnosed with refractory anemia with excess blasts grade II (RAEB-2). A final diagnosis of t-MDS was rendered based on prior radiation history, multilineage dysplasia and unfavorable karyotype. The patient's age and existing co-morbidities pose a significant challenge for the available treatment modalities. The current patient is an unlikely candidate for stem cell transplant which is the only potentially curative modality in MDS.

\section{Discussion}

Establishing a diagnosis of t-MDS vs. de novo MDS is a difficult task. In 50 to $70 \%$ of t-MDS cases, rearrangements in the chromosome 5 and/or 7 are present (6). The average time until onset of t-MDS depends on the type and amount of chemotherapy used. For alkylating agents and topoisomerase inhibitors the average time until onset of t-MDS is 5 to 7 years and 2 to 3 years, respectively. Different chemotherapy agents are associated with different genetic mutations; alkylating agents being associated with rearrangements in chromosome 5 and/or 7 (6-10). Our patient presumably developed t-MDS over 10 years after his treatment with 5-FU and radiation. T-MDS is traditionally associated with alkylating agents and topoisomerase inhibitors. However, cases of 5-FU derived t-MDS have also been reported (11). While HNPCC is not traditionally associated with hematologic malignancies, mutations in mismatch repair genes have been detected in hematologic malignancies and MDS $(4,12,13)$. Also, there are reports of HNPCC families with neurofibromatosis like syndromes and increased incidences of hematologic malignancies (4). A second unrelated primary MDS in a patient with HNPCC is another possibility. While 10 years is longer than the average time until onset of MDS and establishing a definitive etiology may be impossible, the multilineage dysplasia, complex karyotype and history of chemotherapy and radiation are indicative of t-MDS.

Next generation sequencing of the marrow indicated that Muir-Torre syndrome may have played a more important role than chemotherapy/radiation in the pathogenesis of our patient's t-MDS. Our patient's bone marrow had a mutation in SETBP1 p.G870S. As in our patient, SETBP1 p.G870S mutations are associated with monosomy 7 (14). Counterintuitively, while monosomy 7 is associated with t-MDS, SETBPI p.G870S is not commonly associated with t-MDS $(6-10,15)$. Fabiani et al specifically examined SETBP1 mutations in t-MDS and found only $2.83 \%$ (3 of 106) of their therapy related myeloid neoplasm patient cohort had SETBPI point mutations (15). The other mutation detected in our patient was NF1 p.S340Cfs" 12 . NF1 mutations are thought to be causative mutations of neurofibromatosis. Evans et al found NF1 mutations in $97.08 \%$ (166/171) of familial cases of neurofibromatosis type 1 (16). Interestingly, there are reports of HNPCC families with increased incidences of hematologic malignancies and neurofibromatosis like syndromes (4).

Recently a new diagnostic category, 'myeloid neoplasms with germline predisposition', was established by the WHO (5). The genes currently implicated in this category are not involved in HNPCC. However, unlike other hematologic conditions such as chronic myelogenous leukemia, MDS is a cytogenetically heterogeneous disease (17). As more research is conducted, other germline mutations such as those involved in HNPCC, maybe correlated with MDS.

There is an increased frequency of t-MDS in breast cancer survivors who have germline mutations in breast cancer susceptibility genes. One recent study found $21 \%$ (10/41) of breast cancer survivors with t-MDS had inherited mutations in at least one of the following genes: BRCA1, BRCA2, TP53, CHEK2, or PALB2 (6). T-MDS has also been reported to occur at an increased frequency in patients with an inactivating polymorphism in NQO1 gene and the glutathione S-transferase P1 gene $(18,19)$. These genes both produce enzymes responsible for metabolizing toxic byproducts of chemotherapy agents. It has been hypothesized that certain populations may be susceptible to developing t-MDS due to an impaired ability to repair DNA damage or an impaired ability to metabolize chemotherapeutic agents (10).

MTS is caused by an impaired ability to repair DNA damage, but establishing a correlation with t-MDS may be difficult due to MTS's low prevalence. Further studies examining the relationship between t-MDS and MTS are needed. As therapies continue to improve and life expectancies increase, long term complications of chemotherapy and radiation such as t-MDS may become more prevalent. Genetically screening patients undergoing chemotherapy, and maintaining a central database may allow us to further elucidate which patients have an increased risk of developing t-MDS.

\section{References}

1. Lynch HT and de la Chapelle A: Hereditary colorectal cancer. N Engl J Med 348: 919-932, 2003.

2. Wells K and Wise PE: Hereditary colorectal cancer syndromes. Surg Clin North Am 97: 605-625, 2017.

3. Lynch HT, Kimberling W, Albano WA, Lynch JF, Biscone K, Schuelke GS, Sandberg AA, Lipkin M, Deschner EE, Mikol YB, et al: Hereditary nonpolyposis colorectal cancer (lynch syndrome I and II). I. Clinical description of resource. Cancer 56: 934-938, 1985.

4. Bandipalliam P: Syndrome of early onset colon cancers, hematologic malignancies \& features of neurofibromatosis in HNPCC families with homozygous mismatch repair gene mutations. Fam Cancer 4: 323-333, 2005.

5. Arber DA, Orazi A, Hasserjian R, Thiele J, Borowitz MJ, Le Beau MM, Bloomfield CD, Cazzola M and Vardiman JW: The 2016 revision to the world health organization classification of myeloid neoplasms and acute leukemia. Blood 127: 2391-2405, 2016.

6. Churpek JE, Marquez R, Neistadt B, Claussen K, Lee MK, Churpek MM, Huo D, Weiner H, Bannerjee M, Godley LA, et al: Inherited mutations in cancer susceptibility genes are common among survivors of breast cancer who develop therapy-related leukemia. Cancer 122: 304-311, 2016.

7. Larson RA: Etiology and management of therapy-related myeloid leukemia. Hematology Am Soc Hematol Educ Program: 453-459, 2007. 
8. Pedersen-Bjergaard J, Andersen MT and Andersen MK: Genetic pathways in the pathogenesis of therapy-related myelodysplasia and acute myeloid leukemia. Hematology Am Soc Hematol Educ Program: 392-397, 2007.

9. Haase D: Cytogenetic features in myelodysplastic syndromes. Ann Hematol 87: 515-526, 2008.

10. Godley LA and Larson RA: Therapy-related myeloid leukemia. Semin Oncol 35: 418-429, 2008.

11. Park HJ, Choi JH, Lee KA, Kim HC, Nam YS, Oh YH and Lee WS: A case of therapy-related acute myeloid leukemia following 5-fluorouracil chemotherapy. Korean J Intern Med 27: $115-117,2012$.

12. Hangaishi A, Ogawa S, Mitani K, Hosoya N, Chiba S, Yazaki Y and Hirai H: Mutations and loss of expression of a mismatch repair gene, hMLH1, in leukemia and lymphoma cell lines. Blood 89: 1740-1747, 1997.

13. Maeck L, Haase D, Schoch C, Hiddemann W and Alves F: Genetic instability in myelodysplastic syndrome: Detection of microsatellite instability and loss of heterozygosity in bone marrow samples with karyotype alterations. Br J Haematol 109: 842-846, 2000

14. Hou HA, Kuo YY, Tang JL, Chou WC, Yao M, Lai YJ, Lin CC, Chen CY, Liu CY, Tseng MH, et al: Clinical implications of the SETBP1 mutation in patients with primary myelodysplastic syndrome and its stability during disease progression. Am J Hematol 89: 181-186, 2014.

15. Fabiani E, Falconi G, Fianchi L, Criscuolo M, Leone G and Voso MT: SETBP1 mutations in 106 patients with therapy-related myeloid neoplasms. Haematologica 99: e152-e153, 2014.
16. Evans DG, Bowers N, Burkitt-Wright E, Miles E, Garg S, Scott-Kitching V, Penman-Splitt M, Dobbie A, Howard E, Ealing J, et al: Comprehensive RNA analysis of the NF1 gene in classically affected NF1 affected individuals meeting NIH criteria has high sensitivity and mutation negative testing is reassuring in isolated cases with pigmentary features only. EBioMedicine 7: 212-220, 2016.

17. Haase D, Germing U, Schanz J, Pfeilstöcker M, Nösslinger T, Hildebrandt B, Kundgen A, Lübbert M, Kunzmann R, Giagounidis AA, et al: New insights into the prognostic impact of the karyotype in MDS and correlation with subtypes: Evidence from a core dataset of 2124 patients. Blood 110: 4385-4395, 2007.

18. Allan JM, Wild CP, Rollinson S, Willett EV, Moorman AV, Dovey GJ, Roddam PL, Roman E, Cartwright RA and Morgan GJ Polymorphism in glutathione S-transferase P1 is associated with susceptibility to chemotherapy-induced leukemia. Proc Natl Acad Sci USA 98: 11592-11597, 2001.

19. Larson RA, Wang Y, Banerjee M, Wiemels J, Hartford C, Le Beau MM and Smith MT: Prevalence of the inactivating 609C-> T polymorphism in the $\mathrm{NAD}(\mathrm{P}) \mathrm{H}$ :quinone oxidoreductase (NQO1) gene in patients with primary and therapy-related myeloid leukemia. Blood 94: 803-807, 1999. 